OPEN ACCESS

Edited by:

Guo-Chang Fan,

University of Cincinnati, United States

Reviewed by:

Fei Tu,

University of Pittsburgh, United States

Kun Yang,

East Tennessee State University,

United States

Xia Zhang,

Zhejiang University, China

*Correspondence:

Jian-Guang Wang

wjg@wmu.edu.cn

Sheng-Wei Jin

jsw@wmu.edu.cn

Specialty section:

This article was submitted to

Inflammation,

a section of the journal

Frontiers in Immunology

Received: 01 March 2021 Accepted: 06 April 2021

Published: 26 April 2021

Citation:

Yang Y, Li X-Y, Li L-C, Xiao J, Zhu Y-M, Tian $Y$, Sheng $Y-M$,

Chen $Y$, Wang J-G and Jin S-W

(2021) $\gamma \delta$ T/Interleukin-17A Contributes to the Effect of Maresin Conjugates in Tissue Regeneration

1 on Lipopolysaccharide-

Induced Cardiac Injury.

Front. Immunol. 12:674542.

doi: 10.3389/fimmu.2021.674542

\section{$\gamma \delta$ T/Interleukin-17A Contributes to the Effect of Maresin Conjugates in Tissue Regeneration 1 on Lipopolysaccharide-Induced Cardiac Injury}

\author{
Yi Yang ${ }^{1}$, Xin-Yu Li ${ }^{1}$, Lin-Chao $L i^{1}$, Ji Xiao ${ }^{1}$, Yin-Meng Zhu ${ }^{1}$, Yang Tian ${ }^{1}$, \\ Yong-Mao Sheng ${ }^{1}$, Yan Chen ${ }^{1}$, Jian-Guang Wang ${ }^{1,2 *}$ and Sheng-Wei Jin ${ }^{1 *}$
}

\footnotetext{
${ }^{1}$ Department of Anesthesia and Critical Care, The Second Affiliated Hospital and Yuying Children's Hospital of Wenzhou Medical University, Wenzhou, China, ${ }^{2}$ Department of Biochemistry, School of Basic Medical Sciences, Wenzhou Medical University, Wenzhou, China
}

The mechanisms underlying sepsis-induced cardiomyopathy (SIC) remain poorly understood, and there are no specific therapeutics for SIC. We investigated the effects of maresin conjugates in tissue regeneration 1 (MCTR1) on SIC and explored its potential mechanisms. The experiments were conducted using an endotoxemia model induced by lipopolysaccharide (LPS). Mice were given MCTR1 intravenously $6 \mathrm{~h}$ after LPS stimulation. Echocardiography was performed to assess cardiac function $12 \mathrm{~h}$ after LPS administration. Treatment with MCTR1 significantly enhanced cardiac function and reduced LPS-induced increase of mRNA expression levels of inflammation cytokines. Transcriptomic analysis indicated that MCTR1 inhibited neutrophil chemotaxis via the IL17 signaling pathway. We confirmed that MCTR1 reduced the expressions of neutrophil chemoattractants and neutrophil infiltration in the LPS-stimulated hearts. MCTR1 also resulted in a considerable reduction in IL-17A production mainly derived from $\gamma \delta$ T cells. Moreover, our results provided the first evidence that neutralizing IL-17A or depletion of $\gamma \delta$ T cells markedly decreased neutrophil recruitment and enhanced cardiac function in LPSinduced cardiac injury. These results suggest that MCTR1 alleviates neutrophil infiltration thereby improves cardiac function in LPS-induced cardiac injury via the IL-17 signaling pathway. Thus, MCTR1 represented a novel therapeutic strategy for patients with SIC.

Keywords: neutrophil, interleukin-17A, lipopolysaccharide, cardiac injury, $\gamma \delta \mathrm{T}$ cells

\section{INTRODUCTION}

Sepsis is defined as a lethal organ dysfunction caused by a dysregulated host response to infection (1). The heart is one of the most vulnerable organs in sepsis. Sepsis-induced cardiac dysfunction, also called sepsis-induced cardiomyopathy (SIC), has been summarized as a global (systolic and diastolic) but reversible dysfunction of both the left and right sides of the heart (2-4). Despite remarkable 
scientific and clinical efforts, the mechanisms underlying the myocardial dysfunction in sepsis are still not fully understood, and there are no specific therapeutics for SIC (3).

The pathophysiologic cascade of sepsis begins when the host immune system responds to an invading pathogen, resulting in the immune response activation. Interleukin-17A (IL-17A) is one of the members of the IL-17 family. Compared with other members, IL$17 \mathrm{~A}$ plays a more prominent role in the mammalian immune system (5-7). IL-17A is a critical mediator of neutrophil recruitment and migration through the induction of granulopoiesis and the production of neutrophil chemokines, including granulocyte colony-stimulating factor (G-CSF), chemokine (C-X-C motif) ligand 1 protein (CXCL1), and chemokine (C-X-C motif) ligand 2 protein (CXCL2) (8). Although IL-17A exerts a host-defensive role in many infectious diseases, it promotes inflammatory pathology in auto-immunity and other settings (9). Dysregulated IL-17A production or uncontrolled response to IL-17A signaling promotes pathogenic inflammation (10). In the mouse model of myocardial ischemiareperfusion injury, IL-17A was mainly produced by $\gamma \delta \mathrm{T}$ cells, and blockade of IL-17A significantly reduced neutrophil infiltration in the heart and alleviated cardiac injury $(11,12)$. Moreover, anti-IL$17 \mathrm{~A}$ could protect the lungs in lipopolysaccharide (LPS)-induced acute lung injury and improve survival in polymicrobial sepsis induced by cecal ligation and puncture (CLP) (13-15). However, the role of IL-17A in sepsis-induced cardiac dysfunction is not clear.

Specialized pro-resolving mediators (SPMs) are enzymatically derived from essential fatty acids and have crucial roles in restoring tissue homeostasis during tissue inflammation (16). SPMs are distinct from immunosuppressive molecules as they not only dampen inflammation but also promote host defense (17). SPMs are partly defined by their overlapping functions as limiting neutrophil tissue accumulation, counter-regulating proinflammatory cytokines, and encouraging macrophage phagocytosis (18). Previous investigations indicated that the IL17 signaling pathway might involve the inflammation resolving work of SPMs in myocardial infarction and allergic airway inflammation (19-21). In our previous study, we found that maresin conjugates in tissue regeneration 1 (MCTR1), a newly identified SPM, could reduce lipopolysaccharide (LPS)-induced cardiac injury by upregulating mitochondrial biosynthesis and improve the survival rate (22). The mechanism of SPMs on sepsisinduced cardiac dysfunction is not clear, and whether the IL-17 signaling pathway is engaged is also unknown. Therefore, in this study, we tried to clarify the mechanism by which MCTR1 reversed sepsis-induced cardiac dysfunction. We also verified the role of IL-17A in sepsis-induced cardiac dysfunction and confirmed whether IL-17A participated in the effect of MCTR1 on sepsis-induced cardiac dysfunction.

\section{MATERIALS AND METHODS}

\section{Animals}

All animal care and experimental protocols complied with the Guide for the Care and Use of Laboratory Animals of the National Institutes of Health (NIH Publication 8th edition,
2011). Eight-to-twelve-week-old male C57BL/6 mice (Shanghai Experimental Animal Center of China) were used in this study, and the weight of mice was around $25 \mathrm{~g}$. All these mice were housed at four per cage and maintained in a specific pathogenfree room with controlled temperature $\left(23 \pm 1^{\circ} \mathrm{C}\right)$ and humidity (55 $\pm 5 \%)$ under a $12 \mathrm{~h}$ light/dark cycle. The mice were given standard laboratory chow and water ad libitum. All animal experiments were approved by the Animal Studies Ethics Committees of the Second Affiliated Hospital of Wenzhou Medical University.

\section{Experimental Procedures}

To evaluate the effects of MCTR1 on cardiac after LPS stimulation, the mice were randomly divided into four groups: saline control, LPS, LPS plus MCTR1, and MCTR1 along groups. The mouse model of endotoxemia was induced by an intraperitoneal injection of $0.2 \mathrm{ml}$ of sterile saline containing LPS (10 mg/kg, serotype 055: B5; Sigma, Saint Louis, MO, USA). The control mice received an injection of saline in the same volume and route. MCTR1 was obtained from Cayman Chemical (Ann Arbor, MI, USA). MCTR1 was dissolved in ethanol supplied by the manufacturer and was stored at $-80^{\circ} \mathrm{C}$. Ethanol was blown away by nitrogen before use, and MCTR1 was dissolved rapidly in sterile saline to the desired concentration. In the MCTR1 groups, mice received MCTR1 (0.15 nmol/mouse) intravenously via the caudal vein $6 \mathrm{~h}$ after LPS stimulation as previously described (22). The dose of MCTR1 was selected based on previous studies $(22,23)$.

To evaluate the role of the $\gamma \delta$ T cells and IL-17A in the cardiac dysfunction after LPS stimulation, neutralization of endogenous IL-17A and depletion of $\gamma \delta \mathrm{T}$ cells were performed. Neutralization of endogenous IL-17A as previously described (12, 24), $100 \mu \mathrm{g}$ anti-mouse IL-17A antibody (CAT: MAB421, R\&D System, Minneapolis, MN, USA) or $100 \mu \mathrm{g}$ isotype control antibody was administered intravenously $5 \mathrm{~min}$ before LPS treatment. Mice were depleted of $\gamma \delta \mathrm{T}$ cells as previously described $(14,25)$. Five days before treatment with LPS, 500 $\mu \mathrm{g}$ Ultra-LEAFTM Purified anti-mouse TCR $\gamma \delta$ antibody (CAT: 107517, BioLegend, San Diego, CA, USA) was administrated to mice by intraperitoneal injection. Sham depletion mice received equal amounts of isotype control antibodies. For tissue collection, mice were anesthetized by overdose pentobarbital sodium (100 mg/kg intraperitoneal injection) and then sacrificed by bloodletting from the abdominal aorta at $12 \mathrm{~h}$ after LPS treatment.

\section{Echocardiography}

Echocardiography was performed with a Vevo 3100 instrument (Visual Sonics, Toronto, ON, Canada) as described previously $(12,26)$. Mice were anesthetized with $1.2 \%$ isoflurane. Left ventricular end-diastolic volume (LVEDV) and left ventricular end-systolic volume (LVESV) were evaluated using B-mode configuration. Left ventricular ejection fraction (EF) was calculated using the following formula: $E F=[($ LVEDV LVESV)/LVEDV] $\times 100 \%$. Left ventricular end-diastolic diameter (LVEDD) and Left ventricular end-systolic diameter (LVESD) were measured from M-mode tracing. Left ventricular 
fractional shortening (FS) was calculated using the following formula: $\mathrm{FS}=[(\mathrm{LVEDD}-\mathrm{LVESD}) / \mathrm{LVEDD}] \times 100 \%$.

\section{RNA-Seq}

RNA-Seq was performed by Aksomics (Shanghai, China). Significance of differentially expressed genes from transcriptome data was statistically determined with moderated t-test ( $p$-value $<0.05)$, and false discovery rate (FDR; $<0.05 \%)$. The statistically significant genes were submitted to DAVID version 6.8 software (http://david.abcc.ncifcrf.gov) for gene ontology (GO) analysis. Functional pathways were selected in the KEGG database (Kyoto encyclopedia of genes and genomes, https://www.kegg.jp). Significantly enriched GO or functional pathways for up-regulated (UP) and down-regulated (DOWN) groups were visualized with $-\log 10$ transformation of $p$-value.

\section{Western Blotting Analysis}

Tissues from the left ventricular were lysed in a RIPA lysis buffer with PMSF. The supernatants were quantified using the bicinchoninic acid (BCA) method. Then $30 \mu \mathrm{g}$ denatured protein samples were separated by $10-12 \%$ SDSpolyacrylamide gel and transferred onto PVDF membranes (Millipore, Billerica, MA, USA). After blocking with 5\% skimmed milk in TBST at room temperature for $2 \mathrm{~h}$, the membranes were probed overnight at $4^{\circ} \mathrm{C}$ with one of the following primary antibodies: CXCL1 (1:1,000, Affinity Biosciences, Changzhou, China) and G-CSF (1:1,000, Bioss, Beijing, China). The membranes were then washed off of excess antibody and incubated with horseradish peroxidaselinked secondary antibodies $(1: 3,000)$ at room temperature for $1 \mathrm{~h}$. After washing with TBST, the specific bands were visualized using the chemiluminescence detection system and analyzed with the AlphaEaseFC software (Alpha Innotech, San Leandro, CA, USA).

\section{ELISA}

IL-17A in mouse serum was determined using a mouse IL-17A enzyme-linked immunosorbent assay (ELISA) kit (Boyun biotech, Shanghai, China) according to the manufacturer's instructions.

\section{Immunofluorescence}

Immunofluorescence analysis was performed on the paraffinembedded sections. After deparaffinization, rehydration, and antigen retrieval with sodium citrate $(\mathrm{pH} 6.0)$, the tissue sections were blocked with donkey serum at room temperature for $1 \mathrm{~h}$. Subsequently, the tissue sections were incubated with an anti-CXCL1 primary antibody (1:100, Affinity Biosciences, Changzhou, China) or an anti-Ly6G primary antibody (1:100, R\&D Systems, Minneapolis, MN, USA) overnight at $4^{\circ} \mathrm{C}$. Secondary antibodies coupled to Alexa Fluor 594 fluorophores (1:200) were then used and applied for $1 \mathrm{~h}$ at room temperature. Nuclei were stained with DAPI. Finally, tissue sections were observed with a Zeiss fluorescence microscope (Carl Zeiss AG, Oberkochen, Germany). A specific region of interest (ROI) was analyzed using Image (version $1.38 \times, \mathrm{NIH}$, Bethesda, MD, USA) based on previous report (27).

\section{Quantitative PCR}

Total RNA was extracted from mouse left ventricular tissues using TRIzol Reagent (Invitrogen, Carlsbad, CA, USA). Complementary DNA was synthesized from $1 \mu \mathrm{g}$ RNA by reverse transcription kit (Thermo Scientific, Rockford, IL, USA). SYBR Green Real-time PCR Master Mix (Toyobo, Osaka, Japan) was used for real-time PCR. Gene expression levels were normalized with GAPDH as the housekeeping gene, and the expression changes were calculated using the $2^{-\triangle} \triangle \mathrm{Ct}$ method. All primer sequences were summarized in Table $\mathbf{1 .}$

\section{Flow Cytometric Analysis}

Single-cell suspensions were prepared as described previously $(12,28)$. Briefly, mice were deeply anesthetized and intracardially perfused with $20 \mathrm{ml}$ of ice-cold PBS to eliminate blood cells. The hearts were minced with fine scissors and placed into a cocktail of $1 \mathrm{mg} / \mathrm{ml}$ collagenase II (Worthington, Lakewood, NJ, USA), $100 \mathrm{U} / \mathrm{ml}$ elastase (Worthington), and $100 \mathrm{U} / \mathrm{ml}$ DNase I (Sigma-Aldrich) and shaken at $37^{\circ} \mathrm{C}$ for $1 \mathrm{~h}$. Tissue samples were then triturated through a $70 \mu \mathrm{m}$ cell strainer and centrifuged ( $5 \mathrm{~min}, 400 \mathrm{~g}, 4^{\circ} \mathrm{C}$ ). The obtained cells were counted after erythrocyte lysis and washed using PBS for further analysis. For staining, $5 \times 10^{6}$ cells were pre-incubated for $5 \mathrm{~min}$ on ice with anti-CD16/CD32 antibody (2.4G2, BD Bioscience, San Jose, CA, USA) to block the non-specific antibody and then stained with directly conjugated antibodies for $30 \mathrm{~min}$ at $4^{\circ} \mathrm{C}$ in the dark in PBS. For intracellular cytokine staining, single-cell suspensions were stimulated with $50 \mathrm{ng} / \mathrm{ml}$ (PMA, Sigma-Aldrich), $1 \mu \mathrm{g} / \mathrm{ml}$ ionomycin (Sigma-Aldrich), and Golgi-PlugTM (BD Biosciences) for $4 \mathrm{~h}$. Surface staining was performed first. After fixation and permeabilization using

TABLE 1 | Primer sequences for quantitative PCR.

\begin{tabular}{|c|c|c|c|}
\hline Gene & Gene bank no. & Forward (5'-3' sequence) & Reverse ( $5^{\prime}-3^{\prime}$ sequence) \\
\hline Nppb & NM_001287348.1 & GAAGGACCAAGGCCTCACAA & ACTTCAGTGCGTTACAGCCC \\
\hline Tnf & NM_001278601.1 & СССТСАСАСТСACAAACCAC & ACAAGGTACAACCCATCGGC \\
\hline$\| 1 b$ & NM_008361.4 & TGCCACCTITGACAGTGATG & TGATGTGCTGCTGCGAGATT \\
\hline$\| 6$ & NM_031168.2 & TGATGTGCTGCTGCGAGATT & CGCACTAGGTITGCCGAGTA \\
\hline $\mathrm{ccl} 2$ & NM_011333.3 & TGCCCTAAGGTCTTCAGCAC & AAGGCATCACAGTCCGAGTC \\
\hline $\mathrm{ccl} 7$ & NM_013654.3 & GGTCACGCCTAAGGAATGGT & GGGGGAGAATTCTGCAGCTAA \\
\hline $\mathrm{cxcl} 1$ & NM_008176.3 & ACTCAAGAATGGTCGCGAGG & GTGCCATCAGAGCAGTCTGT \\
\hline G-csf & NM_009971.1 & CAGCCCAGATCACCCAGAATC & GCTGCAGGGCCATTAGCTTC \\
\hline$\| 17 a$ & NM_010552.3 & GCTGACCCCTAAGAAACCCC & GAAGCAGTTGGGACCCCTा \\
\hline Gapdh & NM_001289726.1 & GGGTCCCAGCTTAGGTTCAT & GGGACGAGGAAACACTCTCC \\
\hline
\end{tabular}


the Cytofix/Cytoperm Soln kit (BD Biosciences), intercellular proteins were stained. All experiments were performed on an Attune NxT flow cytometer (Invitrogen) and analyzed using FlowJo version 10 software.

\section{Myeloperoxidase Activity}

The heart tissues were weighed and homogenized in the homogenate medium supplied by the myeloperoxidase (MPO) test kit (Jiancheng, Nanjing, China). We determined the MPO activity according to the manufacturer's instructions.

\section{Statistics}

Data are represented as mean \pm standard deviation (SD). All data were analyzed by one-way analysis of variance followed by Tukey's post hoc test for multiple comparisons. $P$-values $<0.05$ were considered statistically significant. Statistical analyses were performed using GraphPad Prism 7.0 software (GraphPad Software, San Diego, CA).

\section{RESULTS}

\section{Post-Treatment With MCTR1 Improved Cardiac Function in LPS-Induced Cardiac Injury}

We previously demonstrated that cardiac function was decreased after LPS administration and peaked at 6 and $12 \mathrm{~h}$ after challenge (22). To verify whether MCTR1 can promote cardiac function recovery from damages of LPS, mice received MCTR1 $6 \mathrm{~h}$ after administration with LPS. Then cardiac function was determined using echocardiography in another $6 \mathrm{~h}$, that is $12 \mathrm{~h}$ after LPS administration (Figure 1A). The results in this study revealed that the left ventricular end-systolic volume (LVESV) significantly increased after the application of LPS. Posttreatment with MCTR1 markedly attenuated the increase of LVESV induced by LPS (Figure 1B). LPS significantly decreased left ventricular fractional shortening (FS) and ejection fraction (EF), which could be notably recuperated by MCTR1 without any effects on baseline cardiac function, and the values of FS and EF in the LPS + MCTR1 group were significantly lower than in the MCTR1 alone group (Figures 1C, D). These results indicated that MCTR1 could partly improve cardiac function in LPS-induced cardiac injury. In accordance with this, MCTR1 significantly reduced the mRNA expression level of natriuretic peptide B (nppb) which was up-regulated by LPS (Figure 1E). MCTR1 treatment markedly attenuated LPS-induced increase of mRNA expression levels of inflammation mediators as well, while the mRNA expressions of those mediators were significantly higher in the LPS + MCTR1 group than in the MCTR1 alone group (Figures 1F-H).

\section{RNA-Seq Analysis}

To explore the mechanism of the effect of MCTR1 on the heart in LPS-induced endotoxemia, we performed RNA sequencingbased transcriptomic profiling of RNA isolated from cardiac tissues derived from LPS group and LPS + MCTR1 group in

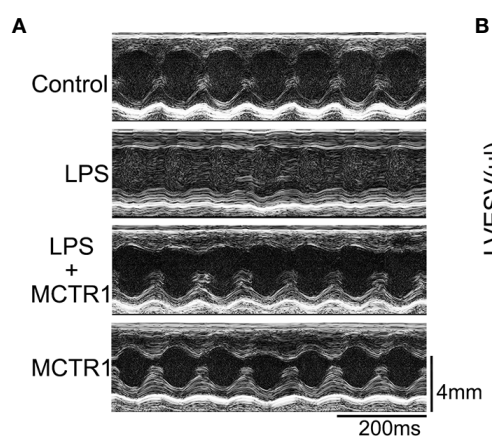

B

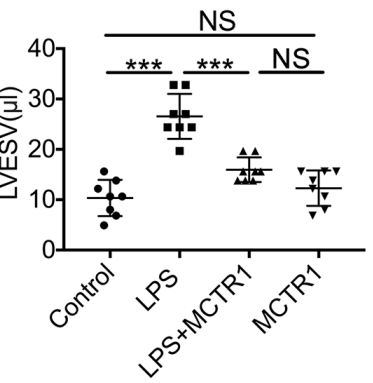

E

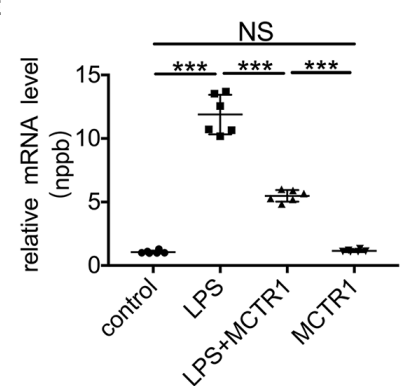

$\mathbf{F}$

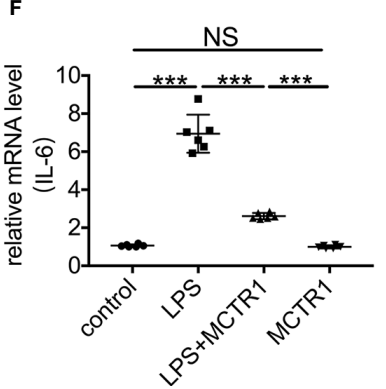

C
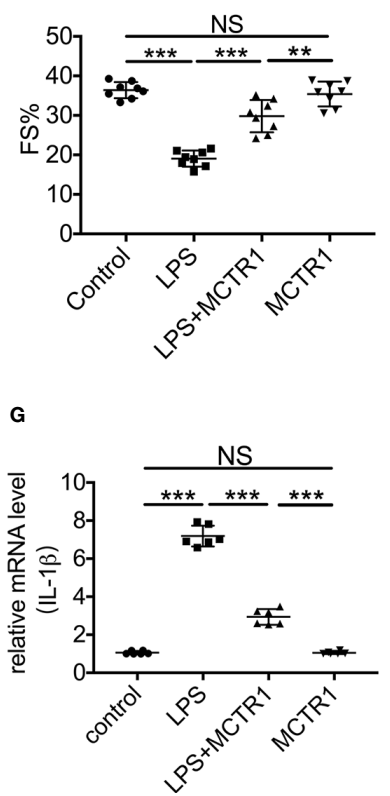
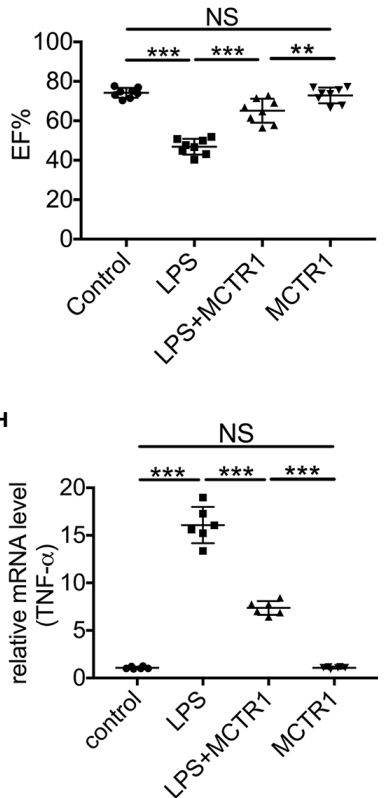

FIGURE 1 | The effect of MCTR1 on cardiac function in LPS-challenged mice. (A) Representative M-mode echocardiographic recordings. (B-D) Left ventricular end-systolic volume (LVESV), fractional shortening (FS), and ejection fraction (EF) were analyzed $(n=8)$. (E-H) Nppb, Tnf- $\alpha$, IL-1 $\beta$, and IL-6 mRNA expressions were examined by quantitative PCR in the hearts $(n=6)$. Data are shown as means \pm SD. ${ }^{\star \star} P<0.01$; ${ }^{\star \star \star} P<0.001$; NS, not significant. 
triplicate. Gene expression profiles of the two groups were evaluated by gene ontology (GO) and functional pathways (Kyoto encyclopedia of genes and genomes, KEGG) analysis. Genes were mostly enriched in the following top five GO groups (Figure 2A): response to organic substance, neutrophil chemotaxis, granulocyte chemotaxis, neutrophil migration and chemokine mediated signaling. KEGG pathway enrichment analysis demonstrated that the IL-17 signaling pathway was the most significantly affected pathway (Figure 2B).

\section{MCTR1 Repressed the Expressions of Neutrophil Chemokines}

We first verified several chemokines that changed significantly in transcriptomic results using quantitative PCR. The mRNA expression levels of CCL2, CCL7, CXCL1, and G-CSF exhibited remarkably enhancement in the LPS group compared with the control group. MCTR1 significantly attenuated the increase of these chemokines induced by LPS. Among them, the expression levels of CXCL1 and G-CSF, well-known neutrophil chemoattractants, changed most obviously (Figures 3A-D). Next, we used western blot to determine the protein expression levels of CXCL1 and G-CSF. The results revealed that MCTR1 dramatically down-regulated the CXCL1 and G-CSF in protein expression levels which were increased in the LPS group, while MCTR1 alone did not affect both of them (Figures 3E-G). Immunofluorescence analyses of cardiac tissues confirmed that CXCL1 mainly expressed in the LPS-injured hearts with very low expression in the non-LPS challenge hearts, and in line with aforementioned results, MCTR1 could considerably lessened the expression of CXCL1 induced by LPS, while the level of CXCL1 was significantly higher in the LPS + MCTR1 group than in the MCTR1 alone group (Figures 3H, I).

\section{MCTR1 Attenuated Neutrophil Infiltration in LPS-Stimulated Hearts}

Consistent with increased chemoattractants, LPS induced a surge in neutrophil recruitment to the myocardium, and MCTR1 dramatically attenuated neutrophil recruitment induced by LPS as determined by flow cytometric analysis of $\mathrm{CD} 11 \mathrm{~b}^{+} \mathrm{Ly}_{6 \mathrm{G}}{ }^{+}$ neutrophils; neutrophil ratio was significantly higher in the LPS + MCTR1 group than in the MCTR1 alone group (Figures 4A, B). Immunofluorescence analyses of Ly6G expression and MPO activity determination confirmed that neutrophil infiltration was increased in the LPS group versus control group, and MCTR1 significantly reduced neutrophil infiltration in the LPS-stimulated hearts, Ly6G expression and MPO activity were significantly higher in the LPS + MCTR1 group than in the MCTR1 alone group (Figures 4C-E).

\section{MCTR1 Alleviated the Expression of IL-17A in LPS-Stimulated Hearts}

As the IL-17 signaling pathway may be involved in the effect of MCTR1 on cardiac function according to the transcriptomic analysis, and one of the prominent roles of IL-17A is neutrophil

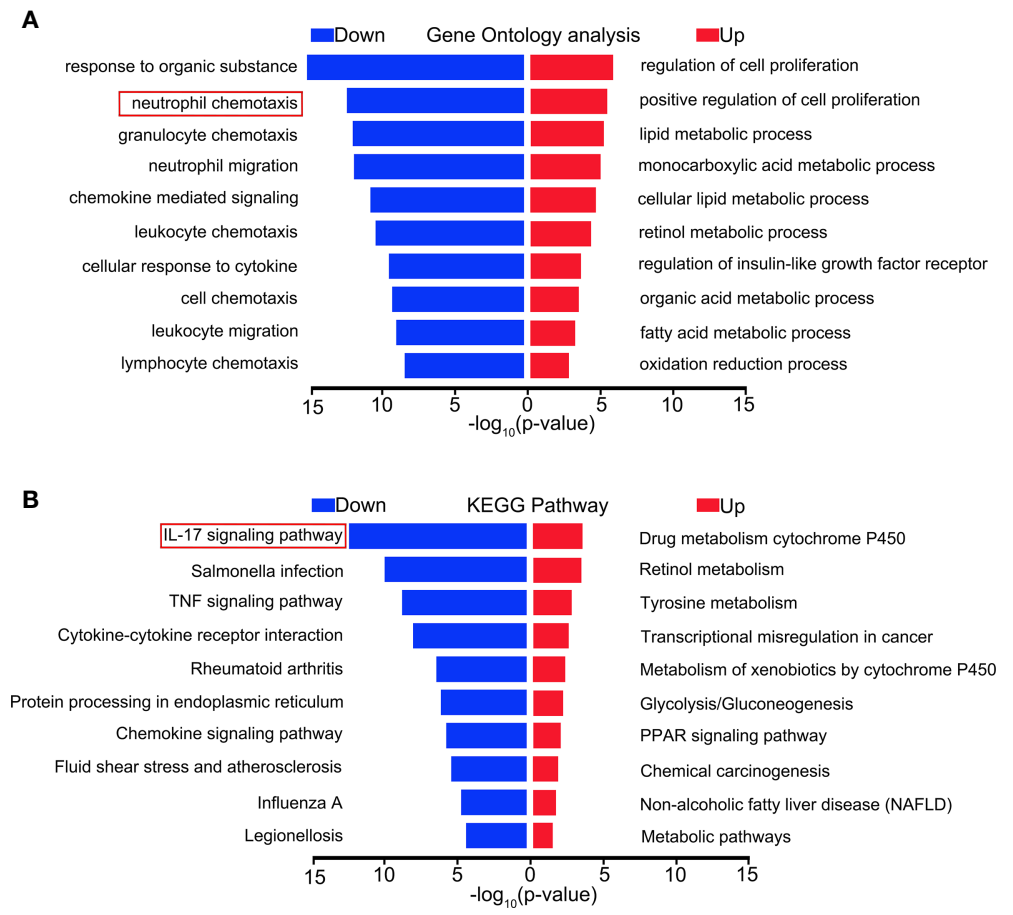

FIGURE 2 | Distinct transcriptional signature between LPS and LPS + MCTR1 groups. $12 \mathrm{~h}$ after LPS treatment, the hearts were harvested and the transcriptome were analyzed by RNA Sequencing (RNA-Seq) technology between LPS and LPS + MCTR1 groups. Significance of differentially expressed genes was divided into UP- and DOWN-regulated gene lists. UP and DOWN genes were submitted for gene ontology (GO) analysis (A) and Kyoto encyclopedia of genes and genomes (KEGG) pathway enrichment analyses (B). 
A

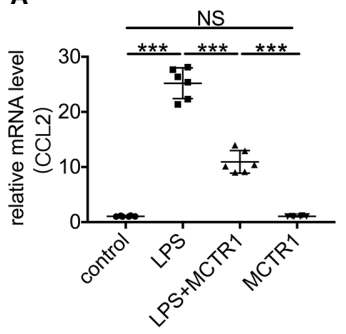

E

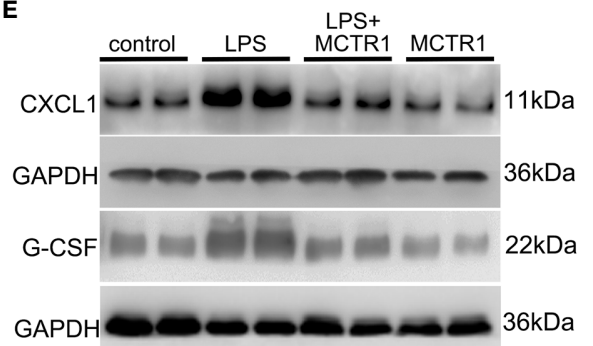

H

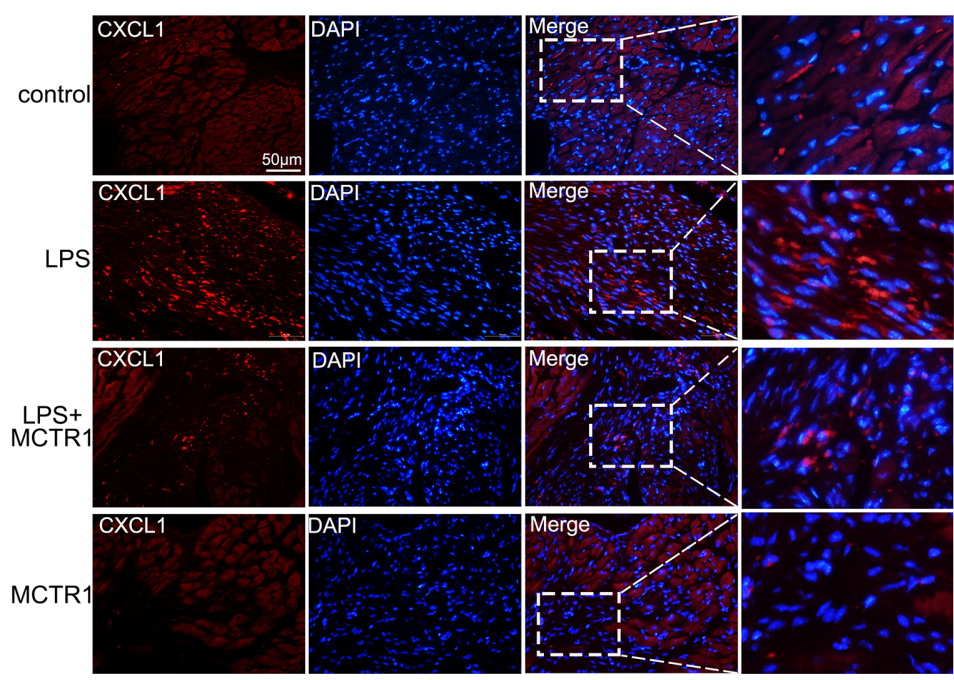

C

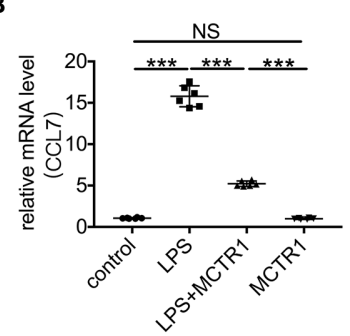

F

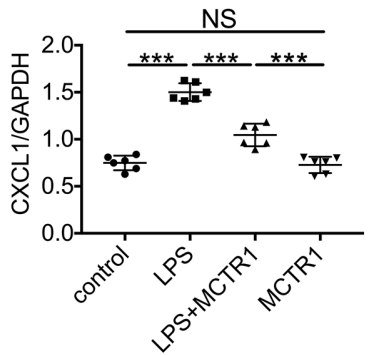

D

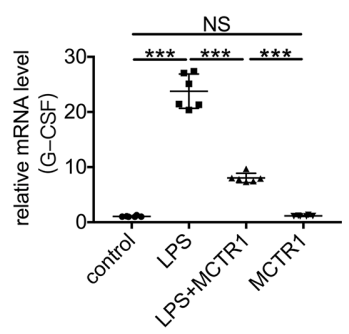

G

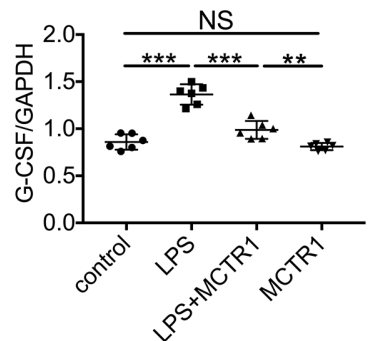

I

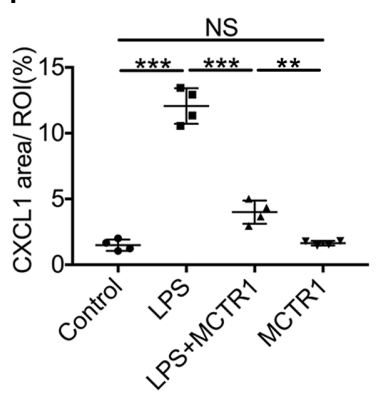

FIGURE 3 | The effect of MCTR1 on chemokines expression in cardiac injury after LPS administration. (A-D) mRNA expression levels of CCL2, CCL7, CXCL1 and G-CSF were examined using quantitative PCR in the hearts $(n=6)$. (E-G) Protein expression levels of CXCL1 and G-CSF were detected by western blotting. Densitometry ratios of target proteins to loading control GAPDH were obtained $(n=6)$. (H) The Representative immunofluorescence staining of CXCL1 in the hearts. (I) Fluorescence intensity of CXCL1 was determined by using Image $J$ software $(n=4)$. Data are shown as means $\pm S D$. ${ }^{\star \star} P<0.01$; ${ }^{* \star *} P<0.001$; NS, not significant.

recruitment, we therefore first determined the expression of IL$17 \mathrm{~A}$ by quantitative PCR and ELISA. The results revealed that LPS stimulation resulted in a significant increase in IL-17A production in the mouse hearts, which was markedly reduced in LPS + MCTR1 group, and the production of IL-17A was significantly higher in the LPS + MCTR1 group than in the MCTR1 alone group (Figures 5A, B). Accumulating evidence demonstrated that lymphocytes and innate myeloid immune cells are able to produce IL-17A (6). To clarify the cell source of IL-17A in the LPS-stimulation hearts, cardiac single cell suspension was stained and analyzed by flow cytometry. The results showed that $\gamma \delta$ T cells were the dominant cells secreting IL-17A, but not CD4 ${ }^{+}$(Th17) or $\mathrm{CD}^{+} \mathrm{T}$ cells (Figures 5C-E). Treatment with MCTR1 resulted in a considerable reduction in IL-17A production from T cells in LPS challenged hearts, and IL17A production was significantly higher in the LPS + MCTR1 group than in the MCTR1 alone group (Figures 5F, G). Collectively, these results indicated that MCTR1 alleviated neutrophil infiltration by reducing the production of IL-17A mainly derived from $\gamma \delta$ T cells in LPS-induced cardiac injury. 
A

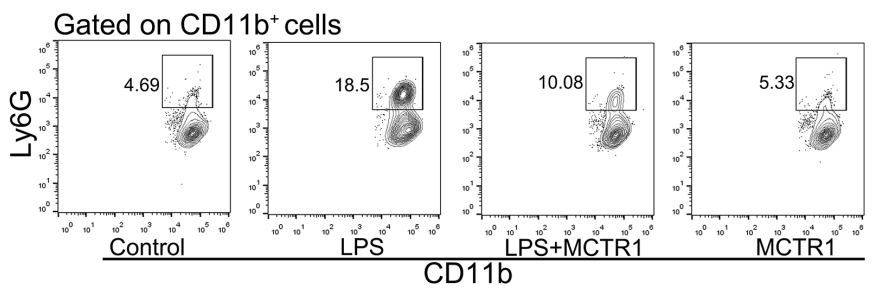

C

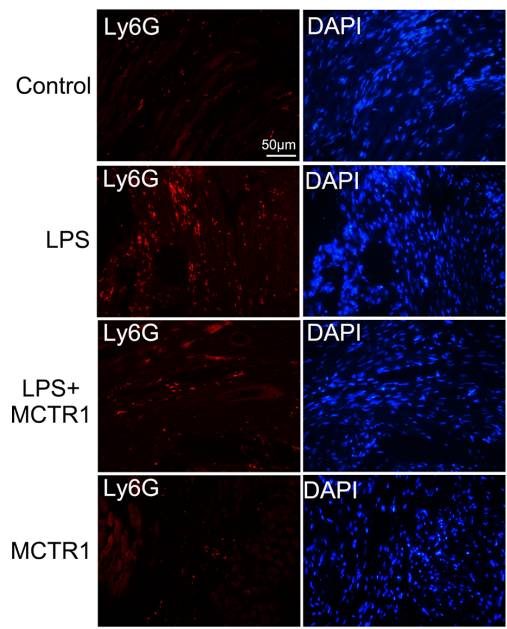

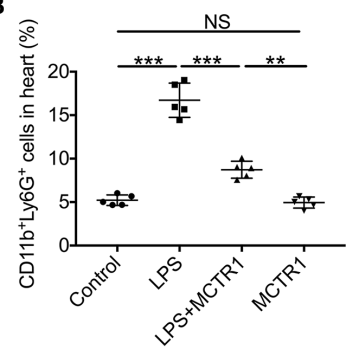

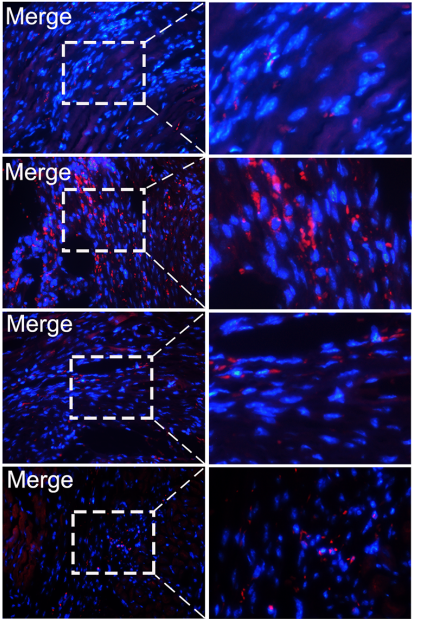

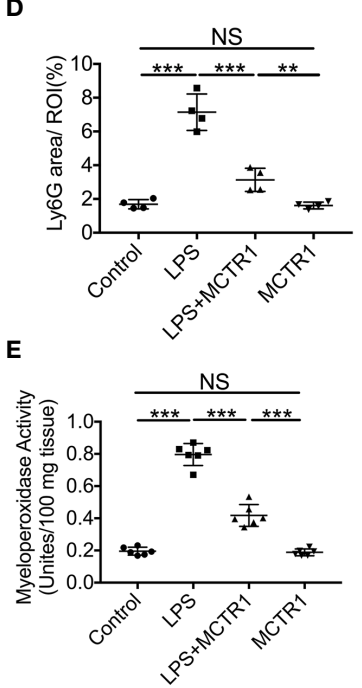

FIGURE 4 | MCTR1 reduced neutrophils infiltration in LPS-induced cardiac injury. (A) The representative flow cytometry dot plots of CD11 $\mathrm{b}^{+}$Ly6G ${ }^{+}$neutrophils infiltrated in myocardium. (B) Percentage of $\mathrm{CD}_{11 \mathrm{~b}}{ }^{+} \mathrm{Ly} 6 \mathrm{G}^{+}$neutrophil population in the hearts $(\mathrm{n}=4)$. (C) Representative immunofluorescence staining of Ly6G in hearts. (D) Fluorescence intensity of Ly6G was determined by Image $\mathrm{J}$ software $(n=4)$. (E) Cardiac MPO activity was examined by MPO test kit $(n=6)$. Data are shown as means $\pm \mathrm{SD}$. ${ }^{\star \star} P<0.01 ;{ }^{\star \star \star} P<0.001$; NS, not significant.

\section{Neutralization of Endogenous IL-17A or Depletion of $\gamma \delta$ T Cells Protects Against LPS-Induced Cardiac Injury}

The roles of IL-17A and $\gamma \delta$ T cells in LPS-induced cardiac injury are still not elucidated, and we have demonstrated that IL-17A was mainly derived from $\gamma \delta \mathrm{T}$ cells in LPS-induced cardiac injury. To verify the effect of IL-17A derived from $\gamma \delta \mathrm{T}$ cells, we first depleted $\gamma \delta$ T cells in mice by administrating anti-TCR $\gamma \delta$ antibody and observed that the production of IL-17A in these $\gamma \delta$ $\mathrm{T}$ cells depletion mice robustly decreased after LPS treatment (Figures 6A, B). We next analyzed mRNA expression levels of neutrophil chemoattractants upon neutralizing IL-17A or depleting $\gamma \delta \mathrm{T}$ cells in endotoxemia. As shown in Figures 6C, D, either neutralization of IL-17A or depletion of $\gamma \delta \mathrm{T}$ cells could significantly reduce mRNA levels of CXCL1 and GCSF. Likewise, neutralizing IL-17A markedly decreased neutrophil infiltration as well as depletion of $\gamma \delta \mathrm{T}$ cells as determined by MPO activity and flow cytometry analysis in LPS-induced cardiac injury (Figures 6E-G). There was no significant difference in CXCL1 and G-CSF mRNA levels, MPO activity, and neutrophil ratio between the LPS + anti-IL$17 \mathrm{~A}$ group and the LPS + anti-TCR $\gamma \delta$ group (Figures $\mathbf{6 C - G}$ ). To assess whether the neutralization of IL-17A or depletion of $\gamma \delta \mathrm{T}$ cells reversed the cardiac function inhibition caused by the general inflammation, we analyzed LVFS and LVEF $12 \mathrm{~h}$ after LPS administration. Neutralization of IL-17A could improve cardiac function to the same extent as depletion of $\gamma \delta$ T cells (Figures 6H-J).

\section{DISCUSSION}

The present study investigated the effects and the mechanisms involved in the post-treatment with MCTR1 in the LPS-induced cardiac injury. Our results revealed that post-treatment with MCTR1 after intraperitoneal injection of LPS enhanced cardiac function and decreased the inflammation mediators in gene and protein levels. According to the transcriptome analysis, we confirmed that MCTR1 caused the reduction of neutrophil chemoattractants levels and the alleviation of neutrophil infiltration. MCTR1 also inhibited the production of IL-17A, which is mainly derived from $\gamma \delta$ T cells in LPS-injured heart. In addition, our study added novel findings that neutralization of IL-17A or depletion of $\gamma \delta \mathrm{T}$ cells significantly ameliorated LPS- 
A

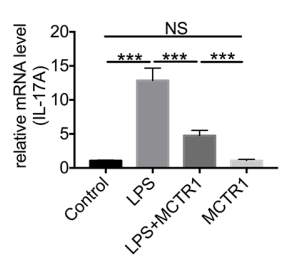

D

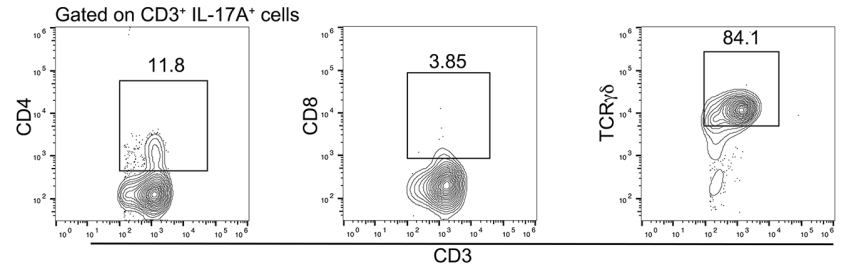

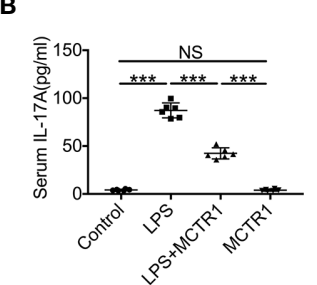

C

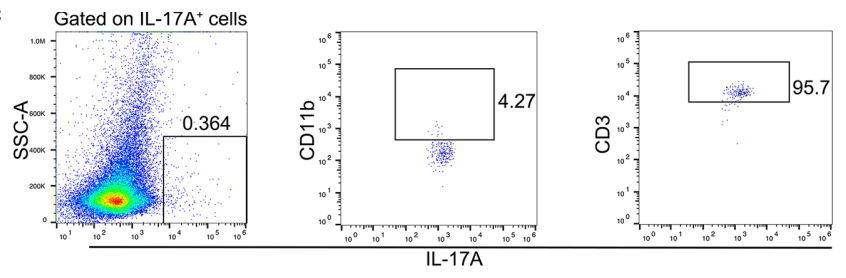

E

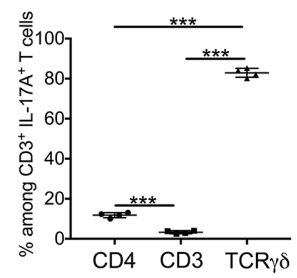

$\mathbf{F}$

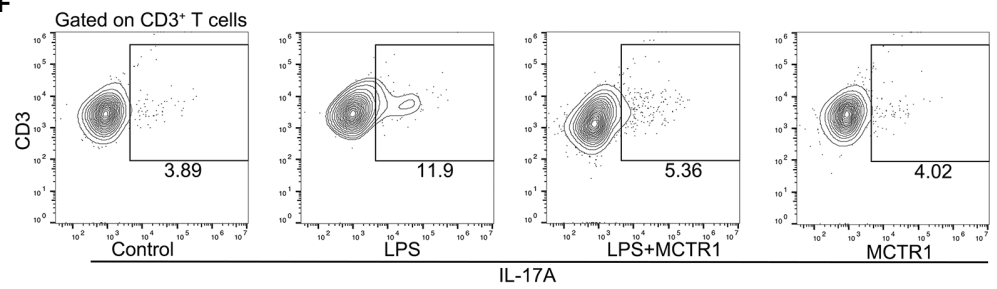

G

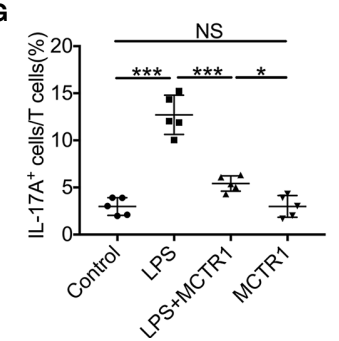

FIGURE 5 | MCTR1 attenuated IL-17A production and IL-17A+ cells in LPS-induced cardiac injury. (A) The mRNA level of IL-17A in hearts was examined using quantitative PCR $(n=6)$. (B) IL-17A in serum was determined by ELISA $(n=6)$. (C) Flow cytometric analysis of CD11b and CD3 expressions on gated IL-17A cells in the LPS-damaged hearts. (D, E) TCR $\gamma \delta$, CD4, and CD8 expressions on gated CD3 ${ }^{+} \mathrm{IL}-17 \mathrm{~A}^{+}$cells were examined by flow cytometric analysis in the LPS-damaged hearts $(n=4)$. $(F, G)$ The percentage of IL-17A cells in all CD3 ${ }^{+} T$ cells was determined by flow cytometric analysis $(n=5)$. Data are shown as means \pm SD. ${ }^{\star} P<0.05 ;{ }^{* \star *} P<0.001 ; \mathrm{NS}$, not significant.

induced cardiac injury, which was associated with a reduction of neutrophil infiltration and improvement of cardiac function.

Patients with SIC typically exhibit ventricular dilatation, reduced ventricular contractility, and/or both right and left ventricular dysfunction with a reduced response to volume infusion $(2,29)$. We previously demonstrated that cardiac function in mice decreased most significantly at 6 and $12 \mathrm{~h}$ after intraperitoneal injection of LPS, and then gradually recovered (22). Here, we found that post-treatment with MCTR1 $6 \mathrm{~h}$ after LPS treatment markedly reduced the left ventricular end-systolic volume and increased the left ventricular fractional shortening, left ventricular ejection fraction. Under this, MCTR1 inhibited LTD4-induced adverse inotropic action in isolated Ciona intestinalis (sea squirt) primordial hearts (23). In the LPS-induced acute lung injury, MCTR1 alleviated lung injury by protecting lung endothelial glycocalyx (30). In an animal model of myocardial infarction (MI), another SPM, RvD1 reduced neutrophil infiltration in ventricular and attenuated inflammation, thereby leading to the improvement of cardiac function (21).

Following cardiac injury, neutrophils lead the first wave of host defense to infection or tissue damage. Neutrophils are essential for the initiation of inflammation, resolving, and cardiac repair $(31,32)$. However, excess infiltration and activation of neutrophils lead to collateral damage in the myocardium (33). After the onset of inflammation, neutrophils and macrophages produce a series of SPMs, including lipoxins, resolvins, protectins, and maresins (16). These SPMs inhibit the excessive infiltration of neutrophils and help orchestrate the return to homeostasis (34-36). Transcriptome profiling in this study suggested that MCTR1 decreased neutrophil infiltration in which the IL-17 signaling pathway involved, and we confirmed that MCTR1 reduced the expression levels of G-CSF and CXCL1 which regulated neutrophil chemotaxis, decreased neutrophil recruitment in LPS-injured heart. IL-17 dominantly signals in non-hematopoietic cells (such as tissue-resident macrophages) to induce chemokines, including CXCL1, CXCL2, and CXCL8 (IL-8). These chemokines can attract neutrophils to infected or injured tissues. In addition, IL-17 induces G-CSF, which promotes the production and maturation of neutrophils from the bone marrow $(33,37)$. Our previous study revealed that MCTR1 promoted M2 polarization of resident macrophages via the STAT6 pathway to accelerate resolution of LPS-induced lung injury (38).

Previous studies revealed that the production of G-CSF and CXCL1 were regulated by IL-17A, which was primarily produced 
A

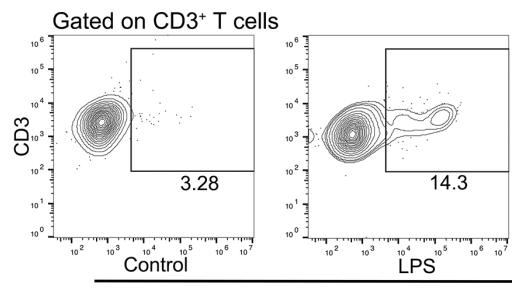

IL-17A
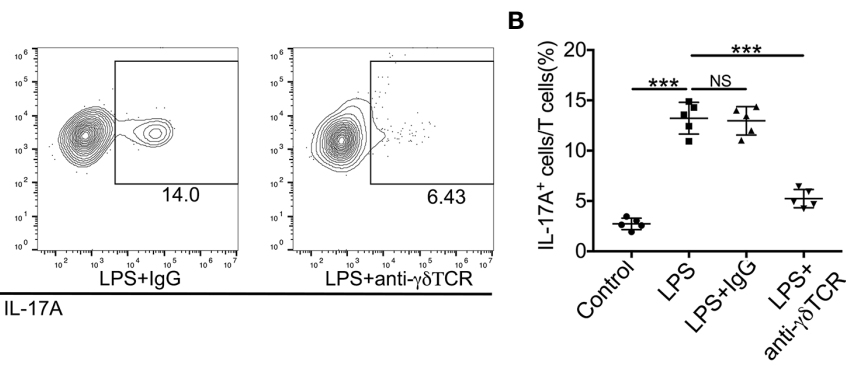

C

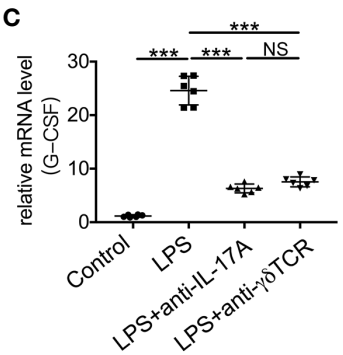

D

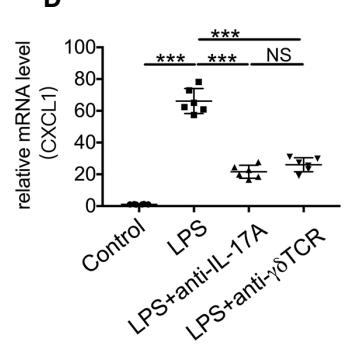

E

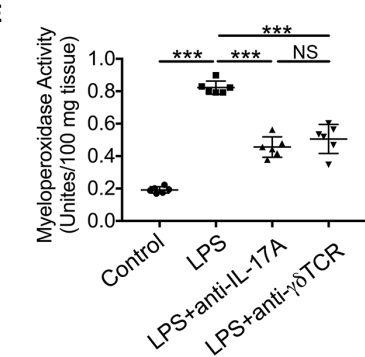

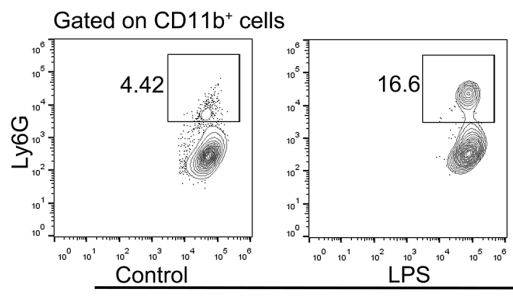

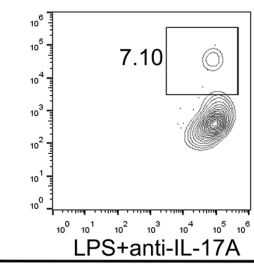

CD11b
H

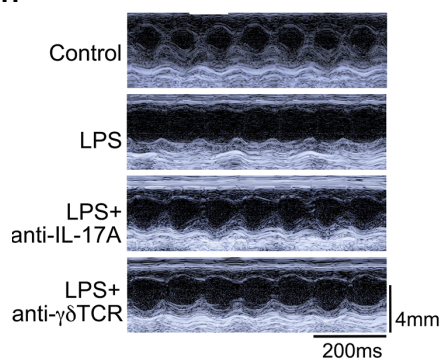

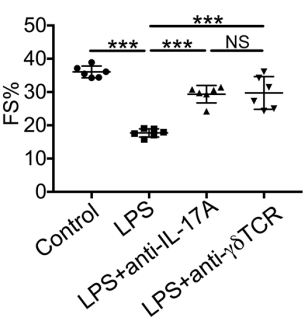

G

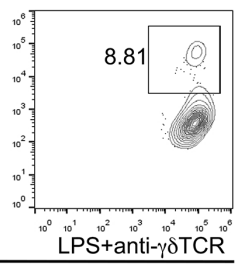

J
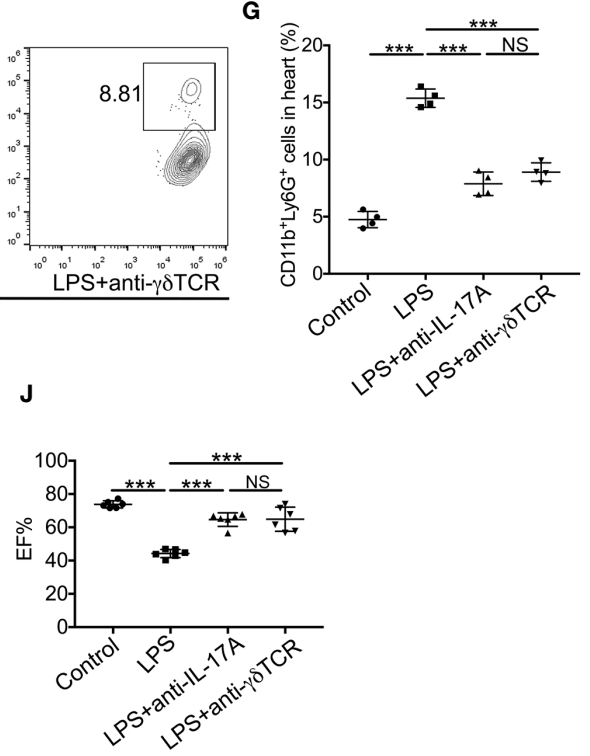

FIGURE 6 | Neutralization of IL-17A or depletion of $\gamma \delta$ T cells reduced neutrophil infiltration and improved cardiac function in LPS-induced cardiac injury Cardiac function was determined using small animal ultrasound and cardiac tissues were collected $12 \mathrm{~h}$ after stimulation with LPS. (A, B) IL-17A ${ }^{+}$cells in all $\mathrm{CD}^{+} T$ cells were examined by flow cytometric analyses $(n=5)$. (C, D) mRNA levels of G-CSF and CXCL1 were analyzed using quantitative PCR $(n=6)$.

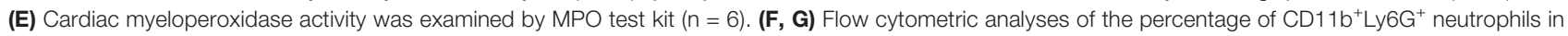
hearts $(n=4)$. (H) Representative cardiographic images of M-mode. (I, J). Left ventricular FS and EF were determined by Echocardiographic analysis $(n=8)$. Data are shown as means \pm SD. ${ }^{* \star} P<0.001$; NS, not significant.

by $\gamma \delta \mathrm{T}$ cells and played a pathogenic role in myocardial $\mathrm{I} / \mathrm{R}$ injury by inducing neutrophil infiltration $(11,12)$. These findings support our results that IL-17A was predominantly produced by $\gamma \delta \mathrm{T}$ cells but not $\mathrm{CD} 4^{+}$or $\mathrm{CD} 8^{+} \mathrm{T}$ cells in LPS-induced cardiac injury. IL-17A also contributed to the mechanisms of cardiac injury in the heart transplantation model $(24,39)$, angiotensin IIinduced hypertensive heart injury (40), and myocarditis-induced cardiac fibrosis (41). The elevated plasma IL-17 level was associated with poor outcomes in post-cardiac arrest syndrome patients and left ventricular diastolic function in patients with diastolic heart failure $(42,43)$. Several clinical trials have been operated to evaluate the correlation between pro-inflammation mediators (IL-17) and sepsis (or SIC). However, the findings have been somewhat inconsistent. In critically ill children with severe sepsis, IL-17 showed a weak positive correlation with severity of illness and was significantly higher among 


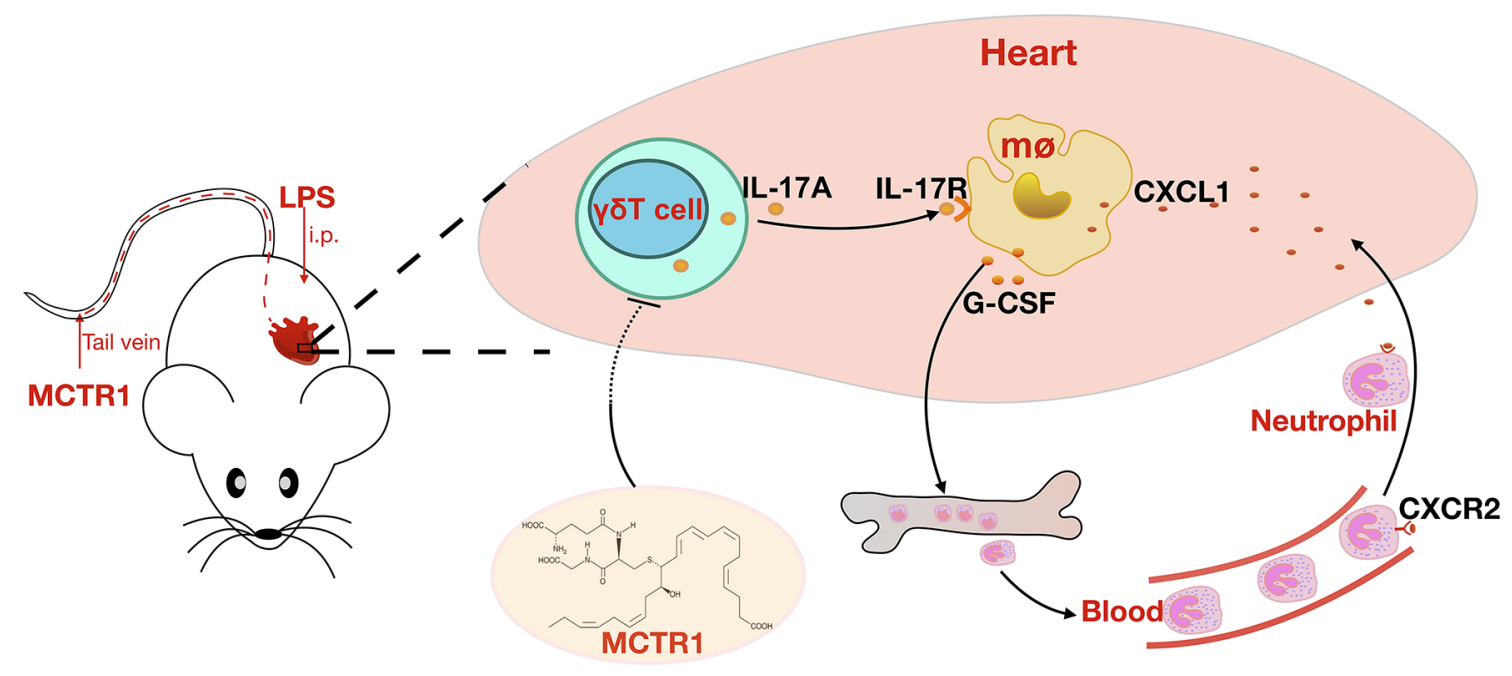

FIGURE 7 | MCTR1 reduces neutrophil infiltration through attenuating IL-17A production derived from $\gamma \delta$ T cells in LPS-induced cardiac injury.

non-survivors (44). Elevated serum IL-17 may increase the susceptibility for septic complications in polytrauma patients (45). However, in another preliminary study, the IL-17/IFN pathway was associated with a faster sepsis resolution and a better survival (46). Here, we found that the level of IL-17A increased in the LPS-stimulated mice. In addition, our results provide the first evidence that neutralization of IL-17A or depletion of $\gamma \delta$ T cells significantly down-regulated the G-CSF and CXCL1 levels, profoundly decreased neutrophil infiltration, and reversed the cardiac function in LPS-induce cardiac injury.

Our results also exhibited that post-treatment with MCTR1 reduced the IL-17A secretion in LPS-induced cardiac injury. Consistent with our results, previous studies have demonstrated that some SPMs could promote inflammation resolution by targeting at IL-17 signaling pathway. For example, resolvin E1 and resolvin E3 alleviated allergic airway inflammation by inhibiting the production of IL-17A $(19,20)$. Maresin 1 reduced IL-17A production by $\gamma \delta \mathrm{TCRmid}^{+}$and $\mathrm{CD} 4^{+} \mathrm{T}$ cells in imiquimod-induced skin inflammation (47). Thus, our findings indicated that IL-17A might get involved in the alternative function of MCTR1 on LPS-induced cardiac injury.

In conclusion, our results provide insights into the protective role of MCTR1 in LPS-induced cardiac injury. MCTR1 alleviated the expression levels of neutrophil chemokines and neutrophil infiltration in the injured heart via the IL-17 signaling pathway (Figures 7). Thus, MCTR1 represented a novel therapeutic strategy for sepsis-induced cardiomyopathy.

\section{DATA AVAILABILITY STATEMENT}

The datasets presented in this study can be found in online repositories. The names of the repository/repositories and accession number(s) can be found in the article/ supplementary material.

\section{ETHICS STATEMENT}

The animal study was reviewed and approved by the Animal Studies Ethics Committees of the Second Affiliated Hospital of Wenzhou Medical University.

\section{AUTHOR CONTRIBUTIONS}

YY and J-GW conceived and designed the study. YY, X-YL, L-CL, $\mathrm{Y}-\mathrm{MZ}$, and YT performed the experiments. JX, YC, and Y-MS analyzed and validated the data. YY, S-WJ, and J-GW reviewed and edited the manuscript. All authors contributed to the article and approved the submitted version.

\section{FUNDING}

This work was supported by the National Natural Science Foundation of China (81870065), Key Research and Development Project of Zhejiang Province (2019c03011) and Major Science and Technology Innovation Project of Wenzhou (2018ZY006).

\section{ACKNOWLEDGMENTS}

YY would especially like to highlight the ongoing and steadfast support of YC. 


\section{REFERENCES}

1. Singer M, Deutschman CS, Seymour CW, Shankar-Hari M, Annane D, Bauer M, et al. The Third International Consensus Definitions for Sepsis and Septic Shock (Sepsis-3). JAMA (2016) 315:801-10. doi: 10.1001/ jama.2016.0287

2. Martin L, Derwall M, Al Zoubi S, Zechendorf E, Reuter DA, Thiemermann C, et al. The Septic Heart: Current Understanding of Molecular Mechanisms and Clinical Implications. Chest (2019) 155:427-37. doi: 10.1016/ j.chest.2018.08.1037

3. Liu YC, Yu MM, Shou ST, Chai YF. Sepsis-Induced Cardiomyopathy: Mechanisms and Treatments. Front Immunol (2017) 8:1021. doi: 10.3389/ fimmu.2017.01021

4. Romero-Bermejo FJ, Ruiz-Bailen M, Gil-Cebrian J, Huertos-Ranchal MJ. Sepsis-Induced Cardiomyopathy. Curr Cardiol Rev (2011) 7:163-83. doi: $10.2174 / 157340311798220494$

5. Veldhoen M. Interleukin 17 is a Chief Orchestrator of Immunity. Nat Immunol (2017) 18:612-21. doi: 10.1038/ni.3742

6. Isailovic N, Daigo K, Mantovani A, Selmi C. Interleukin-17 and Innate Immunity in Infections and Chronic Inflammation. J Autoimmun (2015) 60:1-11. doi: 10.1016/j.jaut.2015.04.006

7. Shimura E, Shibui A, Narushima S, Nambu A, Yamaguchi S, Akitsu A, et al. Potential Role of Myeloid Cell/Eosinophil-Derived IL-17 in LPS-induced Endotoxin Shock. Biochem Biophys Res Commun (2014) 453:1-6. doi: 10.1016/j.bbrc.2014.09.004

8. Xu S, Cao X. Interleukin-17 and its Expanding Biological Functions. Cell Mol Immunol (2010) 7:164-74. doi: 10.1038/cmi.2010.21

9. Patil RS, Bhat SA, Dar AA, Chiplunkar SV. The Jekyll and Hyde Story of IL17Producing Gammadeltat Cells. Front Immunol (2015) 6:37. doi: 10.3389/ fimmu.2015.00037

10. McGeachy MJ, Cua DJ, Gaffen SL. The IL-17 Family of Cytokines in Health and Disease. Immunity (2019) 50:892-906. doi: 10.1016/j.immuni.2019.03.021

11. Liao YH, Xia N, Zhou SF, Tang TT, Yan XX, Lv BJ, et al. Interleukin-17A Contributes to Myocardial Ischemia/Reperfusion Injury by Regulating Cardiomyocyte Apoptosis and Neutrophil Infiltration. J Am Coll Cardiol (2012) 59:420-9. doi: 10.1016/j.jacc.2011.10.863

12. Fan Q, Tao R, Zhang H, Xie H, Lu L, Wang T, et al. Dectin-1 Contributes to Myocardial Ischemia/Reperfusion Injury by Regulating Macrophage Polarization and Neutrophil Infiltration. Circulation (2019) 139:663-78. doi: 10.1161/CIRCULATIONAHA.118.036044

13. Righetti RF, Dos Santos TM, Camargo LDN, Aristoteles L, Fukuzaki S, de Souza FCR, et al. Protective Effects of Anti-IL17 on Acute Lung Injury Induced by LPS in Mice. Front Pharmacol (2018) 9:1021. doi: 10.1183/ 1393003.congress-2017.PA346

14. Flierl MA, Rittirsch D, Gao H, Hoesel LM, Nadeau BA, Day DE, et al. Adverse Functions of IL-17A in Experimental Sepsis. FASEB J (2008) 22:2198-205. doi: 10.1096/fj.07-105221

15. Li J, Zhang Y, Lou J, Zhu J, He M, Deng X, et al. Neutralisation of Peritoneal IL-17A Markedly Improves the Prognosis of Severe Septic Mice by Decreasing Neutrophil Infiltration and Proinflammatory Cytokines. PloS One (2012) 7: e46506. doi: 10.1371/journal.pone.0046506

16. Serhan CN. Pro-Resolving Lipid Mediators are Leads for Resolution Physiology. Nature (2014) 510:92-101. doi: 10.1038/nature13479

17. Basil MC, Levy BD. Specialized Pro-Resolving Mediators: Endogenous Regulators of Infection and Inflammation. Nat Rev Immunol (2016) 16:5167. doi: $10.1038 /$ nri.2015.4

18. Fattori V, Zaninelli TH, Rasquel-Oliveira FS, Casagrande R, Verri WA. Specialized Pro-Resolving Lipid Mediators: A New Class of nonImmunosuppressive and non-Opioid Analgesic Drugs. Pharmacol Res (2020) 151:104549. doi: 10.1016/j.phrs.2019.104549

19. Haworth O, Cernadas M, Yang R, Serhan CN, Levy BD. Resolvin E1 regulates interleukin 23, interferon-gamma and lipoxin A4 to promote the resolution of allergic airway inflammation. Nat Immunol (2008) 9:873-9. doi: 10.1038/ ni. 1627

20. Sato M, Aoki-Saito H, Fukuda H, Ikeda H, Koga Y, Yatomi M, et al. Resolvin E3 Attenuates Allergic Airway Inflammation Via the interleukin-23interleukin-17A Pathway. FASEB J (2019) 33:12750-9. doi: 10.1096/ fj.201900283R
21. Kain V, Ingle KA, Colas RA, Dalli J, Prabhu SD, Serhan CN, et al. Resolvin D1 Activates the Inflammation Resolving Response At Splenic and Ventricular Site Following Myocardial Infarction Leading to Improved Ventricular Function. J Mol Cell Cardiol (2015) 84:24-35. doi: 10.1016/ j.yjmcc.2015.04.003

22. Yang Y, Zhu Y, Xiao J, Tian Y, Ma M, Li X, et al. Maresin Conjugates in Tissue Regeneration 1 Prevents Lipopolysaccharide-Induced Cardiac Dysfunction Through Improvement of Mitochondrial Biogenesis and Function. Biochem Pharmacol (2020) 177:114005. doi: 10.1016/j.bcp.2020.114005

23. Chiang N, Riley IR, Dalli J, Rodriguez AR, Spur BW, Serhan CN. New Maresin Conjugates in Tissue Regeneration Pathway Counters Leukotriene D4stimulated Vascular Responses. FASEB J (2018) 32:4043-52. doi: 10.1096/ fj.201701493R

24. Zhu H, Li J, Wang S, Liu K, Wang L, Huang L. Hmgb1-TLR4-IL-23-IL-17A Axis Promote Ischemia-Reperfusion Injury in a Cardiac Transplantation Model. Transplantation (2013) 95:1448-54. doi: 10.1097/TP.0b013e318293b7e1

25. Maeda Y, Reddy P, Lowler KP, Liu C, Bishop DK, Ferrara JL. Critical Role of Host Gammadelta T Cells in Experimental Acute Graft-Versus-Host Disease. Blood (2005) 106:749-55. doi: 10.1182/blood-2004-10-4087

26. Sun Y, Yao X, Zhang QJ, Zhu M, Liu ZP, Ci B, et al. Beclin-1-Dependent Autophagy Protects the Heart During Sepsis. Circulation (2018) 138:2247-62. doi: 10.1161/CIRCULATIONAHA.117.032821

27. Jensen EC. Quantitative Analysis of Histological Staining and Fluorescence Using Imagej. Anat Rec (Hoboken) (2013) 296:378-81. doi: 10.1002/ar.22641

28. Zouggari Y, Ait-Oufella H, Bonnin P, Simon T, Sage AP, Guerin C, et al. B Lymphocytes Trigger Monocyte Mobilization and Impair Heart Function After Acute Myocardial Infarction. Nat Med (2013) 19:1273-80. doi: 10.1038/ nm.3284

29. Stanzani G, Duchen MR, Singer M. The Role of Mitochondria in SepsisInduced Cardiomyopathy. Biochim Biophys Acta Mol Basis Dis (2019) 1865:759-73. doi: 10.1016/j.bbadis.2018.10.011

30. Li H, Hao Y, Yang LL, Wang XY, Li XY, Bhandari S, et al. MCTR1 Alleviates Lipopolysaccharide-Induced Acute Lung Injury by Protecting Lung Endothelial Glycocalyx. J Cell Physiol (2020) 235:7283-94. doi: 10.1002/ jcp. 29628

31. Epelman S, Liu PP, Mann DL. Role of Innate and Adaptive Immune Mechanisms in Cardiac Injury and Repair. Nat Rev Immunol (2015) 15:117-29. doi: 10.1038/nri3800

32. Potey PM, Rossi AG, Lucas CD, Dorward DA. Neutrophils in the Initiation and Resolution of Acute Pulmonary Inflammation: Understanding Biological Function and Therapeutic Potential. J Pathol (2019) 247:672-85. doi: 10.1002/ path. 5221

33. de Oliveira S, Rosowski EE, Huttenlocher A. Neutrophil Migration in Infection and Wound Repair: Going Forward in Reverse. Nat Rev Immunol (2016) 16:378-91. doi: 10.1038/nri.2016.49

34. Kain V, Halade GV. Role of Neutrophils in Ischemic Heart Failure. Pharmacol Ther (2020) 205:107424. doi: 10.1016/j.pharmthera.2019.107424

35. Buechler C, Pohl R, Aslanidis C. Pro-Resolving Molecules-New Approaches to Treat Sepsis? Int J Mol Sci (2017) 18:476. doi: 10.3390/ijms18030476

36. Ye Y, Zhang HW, Mei HX, Xu HR, Xiang SY, Yang Q, et al. PDX Regulates Inflammatory Cell Infiltration Via Resident Macrophage in LPS-induced Lung Injury. J Cell Mol Med (2020) 24:10604-14. doi: 10.1111/jcmm.15679

37. Schiwon M, Weisheit C, Franken L, Gutweiler S, Dixit A, Meyer-Schwesinger C, et al. Crosstalk Between Sentinel and Helper Macrophages Permits Neutrophil Migration Into Infected Uroepithelium. Cell (2014) 156:456-68. doi: 10.1016/j.cell.2014.01.006

38. Wang Q, Zhang HW, Mei HX, Ye Y, Xu HR, Xiang SY, et al. MCTR1 Enhances the Resolution of Lipopolysaccharide-Induced Lung Injury Through STAT6-mediated Resident M2 Alveolar Macrophage Polarization in Mice. J Cell Mol Med (2020) 24:9646-57. doi: 10.1111/jcmm.15481

39. Oberhuber R, Heinbokel T, Cetina Biefer HR, Boenisch O, Hock K, Bronson RT, et al. Cd11c+ Dendritic Cells Accelerate the Rejection of Older Cardiac Transplants Via Interleukin-17A. Circulation (2015) 132:122-31. doi: 10.1161/CIRCULATIONAHA.114.014917

40. Li Y, Wu Y, Zhang C, Li P, Cui W, Hao J, et al. Gammadeltat Cell-derived interleukin-17A Via an interleukin-1beta-dependent Mechanism Mediates Cardiac Injury and Fibrosis in Hypertension. Hypertension (2014) 64:305-14. doi: 10.1161/HYPERTENSIONAHA.113.02604 
41. Baldeviano GC, Barin JG, Talor MV, Srinivasan S, Bedja D, Zheng D, et al. Interleukin-17A is Dispensable for Myocarditis But Essential for the Progression to Dilated Cardiomyopathy. Circ Res (2010) 106:1646-55. doi: 10.1161/CIRCRESAHA.109.213157

42. Zhuang YG, Chen YZ, Zhou SQ, Peng H, Chen YQ, Li DJ. High Plasma Levels of Pro-Inflammatory Factors interleukin-17 and interleukin-23 are Associated With Poor Outcome of Cardiac-Arrest Patients: A Single Center Experience. BMC Cardiovasc Disord (2020) 20:170. doi: 10.1186/s12872-020-01451-y

43. Xu L, Yan J, Zhang F, Zhou C, Fan T, Chen X, et al. Use of Inflammatory Biomarkers and Real-Time Cardiac Catheterisation to Evaluate the Left Ventricular Diastolic Function in Patients With Diastolic Heart Failure. Heart Lung Circ (2020) 30:396-403. doi: 10.1016/j.hlc.2020.06.017

44. Angurana SK, Bansal A, Muralidharan J, Aggarwal R, Singhi S. Cytokine Levels in Critically Ill Children With Severe Sepsis and Their Relation With the Severity of Illness and Mortality. J Intensive Care Med (2020) 3:885066620912989. doi: 10.1177/0885066620912989

45. Ahmed Ali M, Mikhael ES, Abdelkader A, Mansour L, El Essawy R, El Sayed $\mathrm{R}$, et al. Interleukin-17 as a Predictor of Sepsis in Polytrauma Patients: A Prospective Cohort Study. Eur J Trauma Emerg Surg (2018) 44:621-6. doi: 10.1007/s00068-017-0841-3
46. Razazi K, Boissier F, Surenaud M, Bedet A, Seemann A, Carteaux G, et al. A Multiplex Analysis of Sepsis Mediators During Human Septic Shock: A Preliminary Study on Myocardial Depression and Organ Failures. Ann Intensive Care (2019) 9:64. doi: 10.1186/s13613-019-0538-3

47. Saito-Sasaki N, Sawada Y, Mashima E, Yamaguchi T, Ohmori S, Yoshioka H, et al. Maresin-1 Suppresses Imiquimod-Induced Skin Inflammation by Regulating IL-23 Receptor Expression. Sci Rep (2018) 8:5522. doi: 10.1038/ s41598-018-23623-9

Conflict of Interest: The authors declare that the research was conducted in the absence of any commercial or financial relationships that could be construed as a potential conflict of interest.

Copyright (c) 2021 Yang, Li, Li, Xiao, Zhu, Tian, Sheng, Chen, Wang and Jin. This is an open-access article distributed under the terms of the Creative Commons Attribution License (CC BY). The use, distribution or reproduction in other forums is permitted, provided the original author(s) and the copyright owner(s) are credited and that the original publication in this journal is cited, in accordance with accepted academic practice. No use, distribution or reproduction is permitted which does not comply with these terms. 